\title{
Dynamic pressurization method for measuring permeability and modulus: I. theory
}

\author{
George W. Scherer
}

Published online: 1 November 2006

(C) RILEM 2006

\section{Erratum to: Materials and Structures DOI: 10.1617/s11527-006-9124-x}

Due to an unfortunate turn of events this article was published online June $22^{\text {nd }} 2006$ with two errors in the text. That same version is published on the preceding pages. The below printed paragraphs however are the correct text versions and should be regarded by the reader as the final versions.

This function has been found to provide an excellent fit to stress relaxation data for cement pastes from 1 to 360 days of age [6-8] and for mortar [17]. For the present calculations, we use $\tau_{1}=1.7 \times 10^{9} \mathrm{~s}$ and $\tau_{2}=1.7 \times 10^{4} \mathrm{~s}$, as measured on the one-month old paste. The average relaxation time, found by integrating Equation (58) over time from 0 to $\infty$, is $\tau_{\mathrm{VE}}=2.9 \times 10^{5} \mathrm{~s}$; this is relatively short, probably because the experiment was only continued long enough to relax $\sim 20 \%$ of the stress, so the long-time tail of $\psi$ was not captured. For this type of paste, longer relaxation

The online version of the original article can be found at http://www.dx.doi.org/10.1617/s11527-006-9124-x

G. W. Scherer $(\bowtie)$

Princeton University, Civil \& Env. Eng./PRISM, Eng.

Quad. E-319, Princeton, NJ 08544 USA

e-mail: scherer@princeton.edu runs indicate that $\tau_{\mathrm{VE}}$ exceeds $10^{6} \mathrm{~s}$ within 4 days of curing [7], and continues to rise thereafter; in mortar, relaxation is slower the higher the sand content [17]. To evaluate the influence of VE relaxation, we will evaluate Equation (56) for various values of the ratio

$$
\Lambda=\frac{\tau_{v}}{\tau_{\mathrm{VE}}}
$$

which is a measure of the relative rates of hydrodynamic relaxation (of the pore pressure) and stress relaxation in the porous network.

The transform of $d p_{A} / d \theta$ is $s \tilde{p}_{A}$; substituting these results into Equation (77) and inverting the transform leads to Equation (27).

If the pressure in the chamber is reduced linearly, according to Equation (41), the Laplace transform of the pressure at the exterior surface of the sample is

$$
\tilde{p}_{\mathrm{ext}}=\left(\frac{P_{A}}{s}\right)\left\{1+\frac{1}{s \theta_{0}}\left[\left(1-e^{-s \theta_{d}}\right)-\left(1-e^{-s\left(\theta_{d}+\theta_{0}\right)}\right)\right]\right\}
$$

